\title{
Blood Urea Nitrogen Increased
}

National Cancer Institute

\section{Source}

National Cancer Institute. Blood Urea Nitrogen Increased. NCI Thesaurus. Code

C122438.

Laboratory results demonstrating an increase in the concentration of urea nitrogen in a blood sample. 